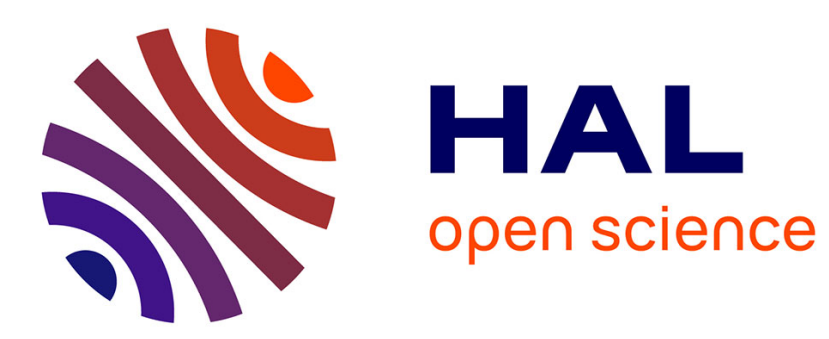

\title{
Dynamic sensing of magnetic nanoparticles in microchannel using GMI technology
}

Kamel Fodil, Matthieu Denoual, Christophe Dolabdjian, M. Harnois, V. Senez

\section{To cite this version:}

Kamel Fodil, Matthieu Denoual, Christophe Dolabdjian, M. Harnois, V. Senez. Dynamic sensing of magnetic nanoparticles in microchannel using GMI technology. IEEE Transactions on Magnetics, 2013, pp.49(1), 124-127. hal-00980710

\section{HAL Id: hal-00980710 https://hal.science/hal-00980710}

Submitted on 18 Apr 2014

HAL is a multi-disciplinary open access archive for the deposit and dissemination of scientific research documents, whether they are published or not. The documents may come from teaching and research institutions in France or abroad, or from public or private research centers.
L'archive ouverte pluridisciplinaire HAL, est destinée au dépôt et à la diffusion de documents scientifiques de niveau recherche, publiés ou non, émanant des établissements d'enseignement et de recherche français ou étrangers, des laboratoires publics ou privés. 


\title{
Dynamic Sensing of Magnetic Nanoparticles in Microchannel Using GMI Technology
}

\author{
K. Fodil ${ }^{1}$, M. Denoual ${ }^{1}$, C. Dolabdjian ${ }^{1}$, M. Harnois ${ }^{2}$, and V. Senez ${ }^{2}$ \\ ${ }^{1}$ GREYC UMR 6072 Université de Caen Basse Normandie et ENSICAEN, 14050 Caen Cedex, France \\ ${ }^{2}$ Université de Lille Nord de France, IEMN, Lille, France
}

\begin{abstract}
This paper presents the detection of magnetic nanoparticles flowing into a microchannel using giant magnetic impedance (GMI) microwire sensor made up of CoFeSiBNb alloy $(40 \mu \mathrm{m}$ diameter and $1 \mathrm{~cm}$ long wire). Detection tests were performed using UltraSmall Particles of Iron Oxyde (USPIO) with diameter of $20 \mathrm{~nm}$. Detection of volume of $180 \mathrm{nl}$ with a concentration of $18 \mathrm{mmol}$ (Fe)/l and flow rate of $260 \mathrm{nl} / \mathrm{s}$ was performed. Such type of measurement can find application in biomedical and clinical diagnosis for in vivo noninvasive detection.
\end{abstract}

Index Terms-Giant magnetoimpedance microwire (GMI), magnetic nanoparticles, microchannel fluidic.

\section{INTRODUCTION}

$\mathbf{M}$ AGNETIC NANOPARTICLES (MNPs) are receiving more and more attention due to their potential applications in biotechnology and biomedical science, such as cell separation, magnetic resonance imaging, controlled drug release and hyperthermia treatments [1]. MNPs can also be used as a label, offering three main advantages compared to fluorescent labels: no-bleaching, detection in opaque media (blood for example) and low toxicity [2].

Whatever the application is, the detection of MNPs is required, to set the intensity of the magnetic field in the case of hyperthermia treatment or to quantify the amount of drug delivered in controlled drug release application. Although depending on the applications, several magnetic sensors can be used for the detection; the detection principle the most commonly used consists in the detection of the magnetization of the MNPs when are exposed to an external magnetic field. The technical challenge of MNPs detection is to develop detection systems that are sensitive enough to detect a single magnetic nanoparticle or clusters of nanoparticles, ideally in continuous flow. Several magnetic sensors, Giant magnetoresistive (GMR) sensors, spin-valve sensors [3], fluxgate, SQUID [4], hall sensors [5] and recently Giant MagnetoImpedance (GMI) sensors have been investigated for the detection of either immobilized MNPs or MNPs flowing in tubes. Depending on the miniaturization and cost constraints on the developed detection system, some sensors are preferred. Specially, the integration of the detection system into a so-called microfluidic microsystem often leads to small sensors such as GMR [6], spin-valve [7] miniaturized hall sensors [8] or GMI [9]-[13].

This paper addresses a new configuration for the setup of a detection system of MNPs flowing into a microfluidic channel in-

\footnotetext{
Manuscript received July 11, 2012; revised August 30, 2012; accepted September 07, 2012. Date of current version December 19, 2012. Corresponding author: K. Fodil (e-mail: kamel.fodil@ensicaen.fr).

Color versions of one or more of the figures in this paper are available online at http://ieeexplore.ieee.org.

Digital Object Identifier 10.1109/TMAG.2012.2218797
}

volving a GMI sensor. The detection principle is described in the first section, then, configuration of the detection system setup is presented. In Section IV, experimental results illustrate the proper functioning of the setup in good agreement with theory. In conclusion, improvements are discussed to quantify the detections limits of such configuration.

\section{Physics PRINCIPLE}

MNPs are small magnetic particles with a size of few tens of nanometers. Their small size makes them superparamagnetic which means that their magnetic susceptibility, $\chi$, is below 1 , and usually they are monodomain. When the MNPs are placed into an external magnetic field $\vec{H}_{\text {magn }}(t)$, a magnetization $\vec{M}$ $(t)$ is induced, expressed for linear homogeneous and isotropic material by

$$
\vec{M}(t)=\chi \vec{H}_{\operatorname{magn}}(t)
$$

The magnetic moment of a volume $V$ of MNPs moving along a $y$-axis induces a magnetic induction $\vec{B}_{n p}(t)$ at a point $P$ of the space according to

$$
\vec{B}_{n p}(t)=\frac{\mu_{0} V}{4 \pi} \underset{\operatorname{grad}}{\longrightarrow}\left(\frac{\vec{M}(t) \cdot \vec{r}}{r^{3}}\right) .
$$

The latter can be expressed by two magnetic field contributions as

$$
B_{x}(t)=\frac{\mu_{0} V|\vec{M}(t)|}{4 \pi} \frac{\left(y_{0}-v_{y} t\right)^{2}-2 x^{2}}{\left(\sqrt{\left(x^{2}+\left(y_{0}-v_{y} t\right)^{2}\right)}\right)^{5}}
$$

and

$$
B_{y}(t)=\frac{\mu_{0} V|\vec{M}(t)|}{4 \pi} \frac{-3 x\left(y_{0}-v_{y} t\right)}{\left(\sqrt{\left(x^{2}+\left(y_{0}-v_{y} t\right)^{2}\right)}\right)^{5}}
$$

where $\mu_{0}$ is the permeability of free space $\left(4 \pi 10^{-7} \mathrm{H} / \mathrm{m}\right), x$ and $y$ depending on the position of $P$ relative to the magnetic sample, $t$ is the time and $v_{y}$ is the speed of the MNPs. 


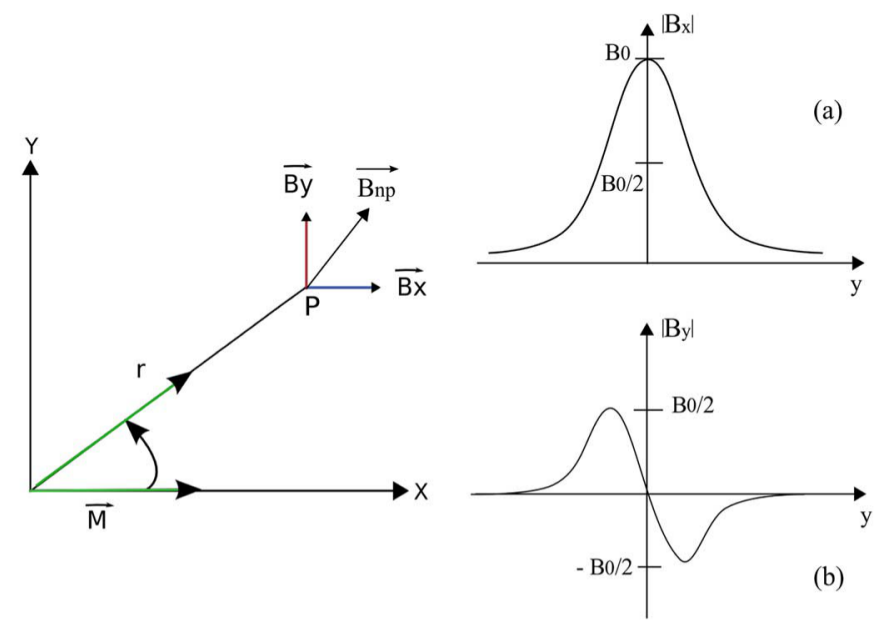

Fig. 1. Representation of the magnetic induction produced by a volume of MNPs in the space at the point $P$. (a) Signal expression of the $B_{x}$ contribution and (b) signal expression of the $B_{y}$ contribution. $\mathrm{B}_{0}$ is a constant.

The illustration of these two field contributions in Fig. 1 exhibits that the maximum of $B_{x}$ is twice higher than the one of $B_{y}$. The detection of the magnetic induction produced by the MNPs can be sensed by a magnetic sensor. The latter is usually placed perpendicularly to the magnetization field $\vec{H}_{\text {magn }}$ in order to sense only the contribution of the magnetic induction produced by the MNPs and not to be saturated by $\vec{H}_{\text {magn }}$. According to the detection setup, two major configurations can be found in literature [10], [14] depending on the directions of the magnetic sensor sensitive axis, the displacement of the MNPs and the magnetization field. Depending on the configuration either the $B_{x}$ or the $B_{y}$ contribution of the magnetic induction $\vec{B}_{\mathrm{np}}(t)$ of the MNPs is predominant. In previous works, configurations favoring $B_{y}$ were investigated in either static [5] or dynamic measurement setups [9], [10]. Configurations favoring $B_{y}$ have also been developed with GMR [14] or SQUID [15] as magnetic sensors.

Here, the measurement of the $B_{x}$ contribution is privileged because of the expected better response. In these conditions, the measured signal is expressed by

$$
\left|B_{m}(t)\right|=\frac{\mu_{0} V|\vec{M}(t)|}{4 \pi} \iint \frac{y(t)^{2}-2 x^{2}}{\left(\sqrt{\left(x^{2}+y(t)^{2}\right)}\right)^{5}} d x d y
$$

with $y(t)=y_{0}-v_{y} t$, where $y_{0}$ is the initial position of MNPs. The simulated signal is given in Fig. 2.

\section{EXPERIMENTAL SETUP}

The experimental setup is depicted in Fig. 3. MNPs are magnetized by a pair of Helmholtz coils in an AC magnetization field. The latter is perpendicular to the sensitive axis of the GMI microwire sensor in order to sense only the tiny stray field from the MNPs which are exposed to the magnetization field. Such arrangement prevents the saturation of the magnetic sensor and easily enables high dynamic range measurements. The frequency of the magnetization field $\left(f_{\text {magn }}\right)$ is selected taking into

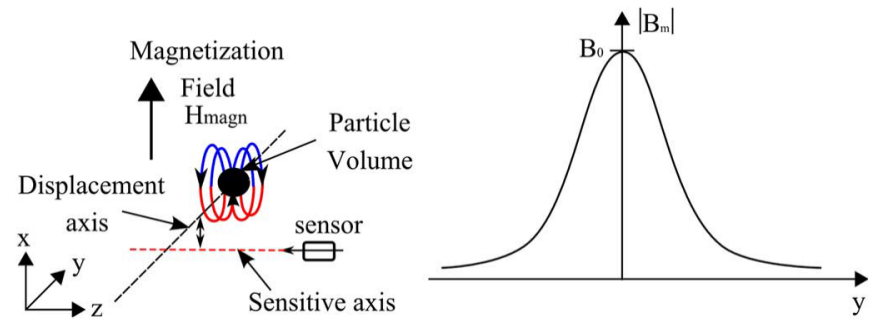

Fig. 2. Simulated signal response as a function of the displacement of the MNPs when the magnetization field is applied on the $x$-axis and the sensitive axis of the GMI microwire is along the $z$-axis which corresponding to the case of the setup of this work. We notice that the sensitive axis of the GMI and the axis of the displacements of the nanoparticles are not coplanar.

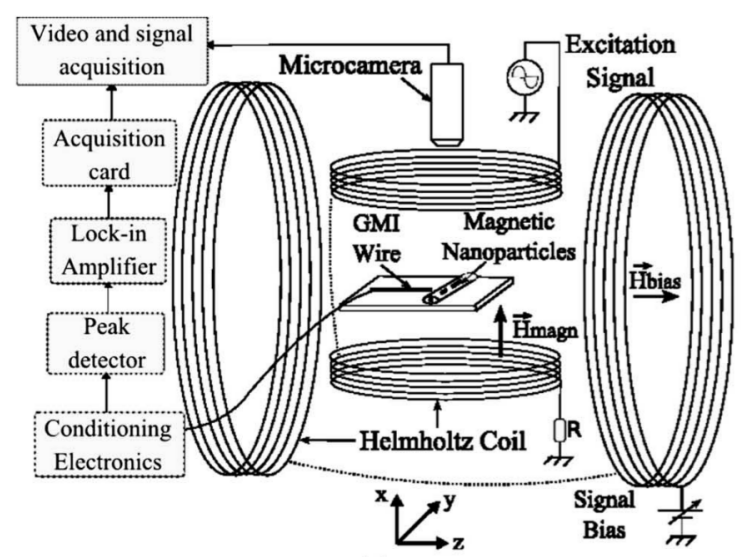

(a)

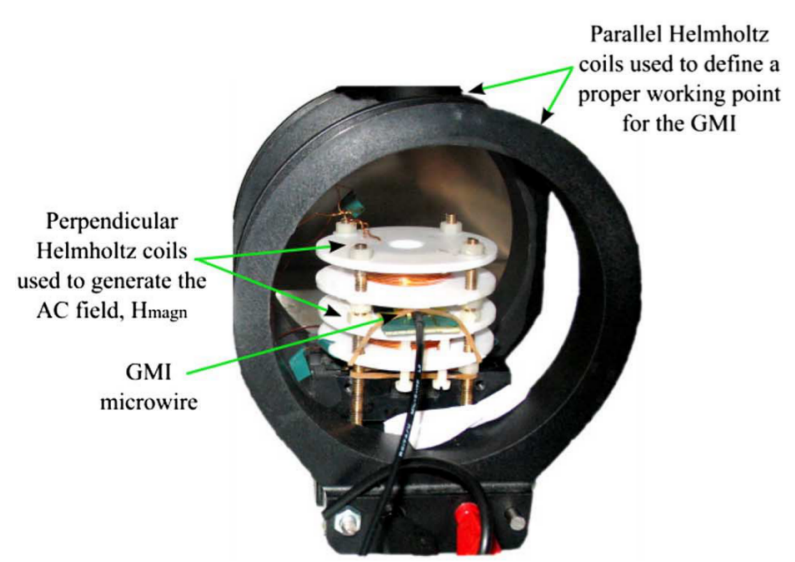

(b)

Fig. 3. (a) Schematic and (b) picture view of the general setup.

account the noise limitation of the GMI microwire and in order to optimize the signal noise ratio (SNR) of the GMI sensor microwire knowing the limited bandwidth of the pair of Helmholtz coils. The magnetization field is applied at a frequency $f_{\text {magn }}$ of $1 \mathrm{kHz}$ with an amplitude of $9200 \mathrm{~A} / \mathrm{m}$. Another set of Helmholtz coils is located parallel to the sensitive axis of the GMI microwire to define a proper working point for the GMI microwire sensor with a DC bias field $H_{\text {bias }}$. The MNPs flow into a glass microchannel placed between the two sets of Helmholtz coils.

The maximum field generated by the MNPs $h_{\mathrm{np}}$ is given by

$$
h_{n p}=\frac{2 \mu_{0} \chi V}{4 \pi d^{3}} \cdot H_{\mathrm{magn}} \cdot \sin \left(2 \pi f_{\mathrm{magn}} t\right)
$$


where $d$ is the mean distance between the MNPs and the sensitive axis of the GMI microwire. The magnetoimpedance signal variation is written such as [16]

$Z\left(\omega_{0}, H_{\text {bias }}+h_{n p}\right)=Z\left(\omega_{0}, H_{\text {bias }}\right)+\left.\frac{\partial Z\left(\omega_{0}, H\right)}{\partial H}\right|_{H=H_{\text {bias }}} \cdot h_{\mathrm{np}}$.

The voltage across the GMI microwire is expressed by

$$
V_{\mathrm{GMI}}(t)=Z\left(\omega_{0}, H_{\mathrm{bias}}+h_{\mathrm{np}}\right) \cdot I_{A C} \cdot \sin \left(\omega_{0} t\right) .
$$

The GMI microwire sensor is connected to a diode rectifier demodulation circuit and then to an amplifier.

So, the output signal is given by

$$
V_{s}(t)=\left(Z\left(\omega_{0}, H_{\text {bias }}\right)+\left.\frac{\partial Z\left(\omega_{0}, H\right)}{\partial H}\right|_{H=H_{\text {bias }}} \cdot h_{n p}\right) \cdot I_{\mathrm{AC}} \cdot G
$$

where $G$ is the gain of the amplifier, $\omega_{0}$ and $I_{A C}$ are the pulsation and current excitation of the GMI sensor, respectively. The frequency $f_{0}$ corresponding to the pulsation excitation of the GMI microwire is $15 \mathrm{MHz}$ and the current is $12 \mathrm{~mA}$.

Thus, the amplitude variations due to the magnetic liquid displacement are demodulated with a lock-in amplifier. At the working point, fixed by $H_{\text {bias }}$, the sensitivity of the GMI microwire $\left(\mathrm{Co}_{70,54} \mathrm{Fe}_{3,99} \mathrm{Si}_{15,91} \mathrm{~B}_{7,9} \mathrm{Nb}_{2,96}\right)[17]$ is $1300 \mathrm{~V} / \mathrm{T}$ and the gain of conditioning electronics $G$ is 500. A National Instrument acquisition card (NI-USB-6251) coupled to a Labview interface is used for the recording of the output of the lock-in amplifier.

Finally, the total output signal after synchronous demodulation can be expressed by

$$
V_{t}=\frac{4}{\pi} \cdot \frac{\mu_{0} \cdot G \cdot G_{d} \cdot T_{r} \cdot V \cdot \chi \cdot H_{\mathrm{magn}}}{4 \pi \cdot d^{3}}
$$

where $G_{d}$ is the gain of the lock-in amplifier and $T_{r}$ is the sensitivity of the GMI at the working point. From there, the signal to noise ratio of the measure can be calculated knowing the equivalent magnetic noise of the system. In the present setup, the noise floor of the GMI microwire is around $150 \mathrm{pT} / \sqrt{ } \mathrm{Hz}$.

\section{EXPERIMENTAL RESULTS}

Before performing dynamic measurements with the MNPs flowing into the microchannel $\left(v_{y} \neq 0\right)$, the MNPs used were characterized. For that, static measurements were performed with different concentrations of UltraSmall Particles of Iron Oxyde (USPIO) provided by the French laboratory Guerbet.

\section{A. Static Measurement $\left(v_{y}=0\right)$}

The experiment is performed by putting a sample of MNPs in the setup and measuring the difference voltage between the signal with and without sample. Fig. 4 shows results of static measurements, for different MNPs concentrations in the same volume of $180 \mu \mathrm{l}$. Nine samples of MNPs were used with decreasing concentration from $18 \mathrm{mmol}(\mathrm{Fe}) / 1$ down to $70 \mu \mathrm{mol}$ $(\mathrm{Fe}) / 1$ corresponding to a $1 / 2$ dilution factor.

The recorded signal illustrated in Fig. 4 is proportional to the concentration, which is in good agreement with theory. The

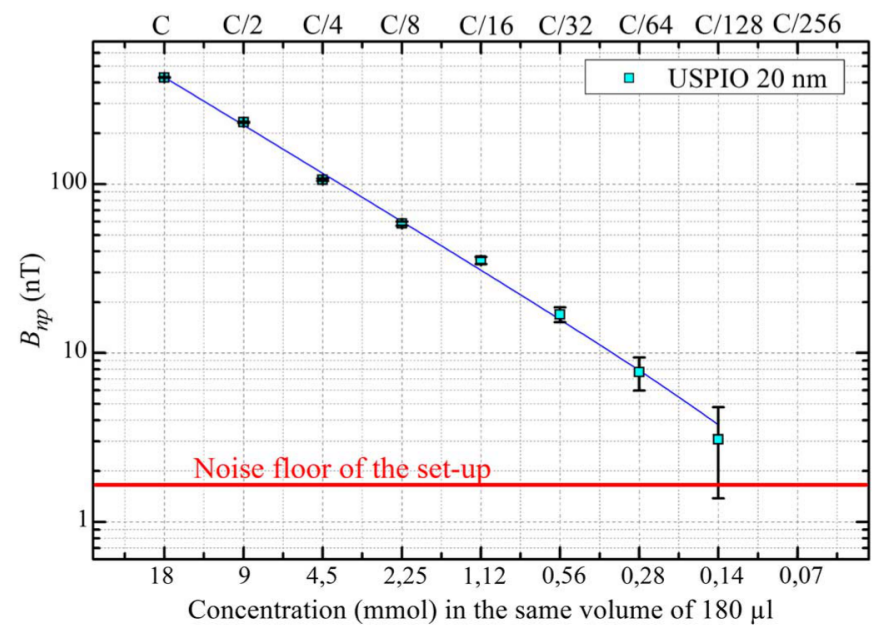

Fig. 4. Sensed magnetic field for different concentrations of MNPs in static measurement. The error given by the RMS variance of the noise is plotted in the graph.

smallest concentration detected was $140 \mu \mathrm{mol}(\mathrm{Fe}) / 1(18 \mathrm{mmol} /$ 128). The SNR limitation of the system prevents the detection of the last sample. The postdetection bandwidth of the lock-in amplifier was set at $1.6 \mathrm{~Hz}$ and the noise level of the GMI microwire at $1 \mathrm{kHz}$ is close to $1 \mathrm{nT} / \sqrt{ } \mathrm{Hz}$. The RMS variance of noise in the bandwidth is $1.7 \mathrm{nT}$, in agreement with SNR depicted in Fig. 4.

\section{B. Dynamic Measurement $\left(v_{y} \neq 0\right)$}

For dynamic measurement, the highest concentration of USPIOs was mixed with oil to form plugs of liquid with MNPs and injected in the microchannel using a syringe. Fig. 5 shows examples of the data collected during a continuous flow experiment.

A video camera and an acquisition card record the displacement of the liquid of MNPs and the data, respectively, at the same time to ensure the correlation between the measured magnetic signal and the position of the plugs of MNPs in the channel. The measured signal rises when the edge of the plugs in the channel approach the sensitive axis of GMI microwire, as shown in Fig. 5. It is maximum when the plug of MNPs is symmetrically apart from the sensitive axis of the GMI. It corresponds to what it is expected by theory (Fig. 2).

Fig. 5(a) and (b) shows representative data acquired during the experiments corresponding to two successive passages of the MNPs liquid and to the passage of the smallest volume sensed, respectively. A graph paper was placed close to the microfluidic channel for a better evaluation of the sample sizes (the square side is $1 \mathrm{~mm}$ ).

The small pictures are extracted from video recording corresponding to the passage of the MNPs. The postdetection bandwidth of the lock-in amplifier was set at $7.8 \mathrm{~Hz}$ and yields to SNR of 3. The smallest volume of MNPs fluid detected during the experiments was $180 \mathrm{nl}$ with a flow rate of $260 \mathrm{nl} / \mathrm{s}$ and a concentration of $18 \mathrm{mmol}(\mathrm{Fe}) / \mathrm{l}$. Taking into account the SNR of the setup, volumes down to $60 \mathrm{nl}$ should be detectable. 


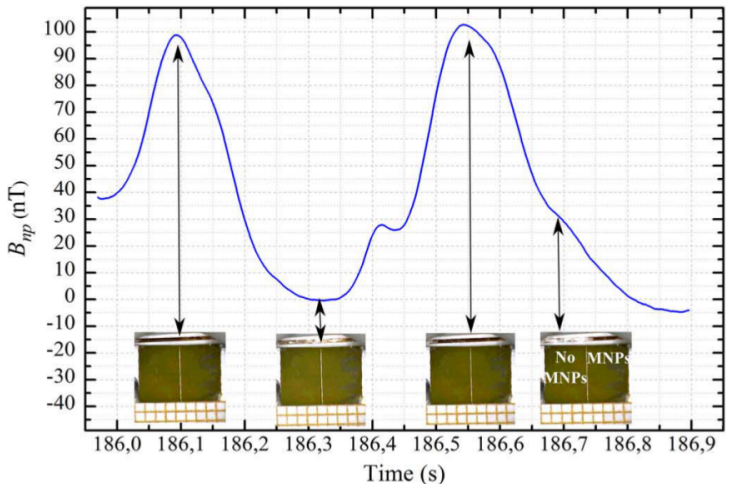

(a)

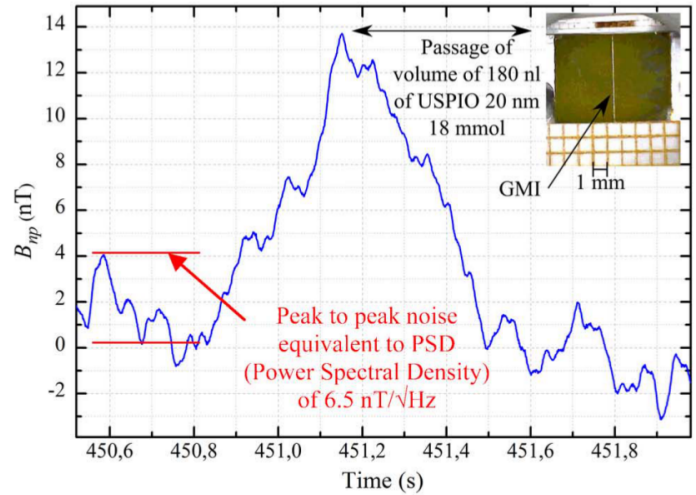

(b)

Fig. 5. Measured magnetic field during the passage of a given volumes of the MNPs in the microchannel. (a) Shows two successive passages of full volume of the microchannel. (b) Shows the smallest volume sensed during the experiments.

\section{Discussion}

Notice that in the case of dynamic measure, the setup bandwidth is adapted to the liquid speed. Nevertheless, it increases the noise of the detection system, and consequently decreases the signal to noise ratio. For the in vivo application of the MNPs, the concentration of MNPs in the human body can't exceed a dosage of $40 \mu \mathrm{mol}(\mathrm{Fe}) / 1$ to avoid MNPs agglomeration in the organism [18]. Thereby, for real times acquisition of liquid flowing, in blood vessel for example, a fast detection system is required. Presently, the noise floor of the GMI was $150 \mathrm{pT} / \sqrt{ } \mathrm{Hz}$. A noise floor less than $1 \mathrm{pT} / \sqrt{ } \mathrm{Hz}$ can be obtained [19]. In this case, and with a voltage source allowing to supply the pair of Helmholtz coils over the corner frequency of the GMI microwire noise, a concentration of MNPs of 150 nmol (Fe)/1 should be detectable in static measurement. It corresponds to a concentration of $20 \mu \mathrm{mol}(\mathrm{Fe}) / 1$ in dynamic detection with a MNPs speed of $4.3 \mathrm{~mm} / \mathrm{s}$. Therefore, an in vivo detection of MNPs flowing in blood vessels is possible.

\section{CONCLUSION}

We have developed a detection system of MNPs flowing into a microchannel and we have successfully sensed the passage of the MNPs. With an optimized GMI microwire sensor and an optimized voltage source, detection of MNPs with concentrations compatible with in vivo experiments becomes achievable, opening the way to new interesting biomedical applications.

\section{ACKNOWLEDGMENT}

The authors would like to thank MXT Montréal for providing the GMI microwires.

\section{REFERENCES}

[1] Q. A. Pankhurst, J. Connolly, S. K. Jones, and J. Dobson, “Applications of magnetic nanoparticles in biomedicine," J. Phys. D: Appl. Phys., vol. 36, pp. 167-181, 2003.

[2] E. Parton, G. Borghs, and R. De Palma, "Biomedical applications using magnetic nanoparticles," Solid State Technol., vol. 50, 2007.

[3] G. Li, V. Joshi, R. L. White, S. X. Wang, J. T. Kemp, C. Webb, R. W. Davis, and S. Sun, "Detection of single micron-sized magnetic bead and magnetic nanoparticles using spin valve sensors for biological applications," J. Appl.Phys., vol. 7557, pp. 10-13, 2012.

[4] M. S. Katsura, T. Yasuda, K. Hirano, A. Mizuno, and S. Tanaka, "Development of a new detection method for DNA molecules," Science Technol., vol. 14, p. 1131, 2001.

[5] L. Ejsing, M. F. Hansen, and A. Menon, "Magnetic microbead detection using the planar Hall effect," J. ournal of Magnet. Magn. Mater., vol. 293, pp. 677-684, 2005.

[6] N. Pekas, M. D. Porter, M. Tondra, A. Popple, and A. Jander, "Giant magnetoresistance monitoring of magnetic picodroplets in an integrated microfluidic system," J. Appl. Phys., vol. 85, p. 4783, 2004.

[7] H. A. Ferreira, N. Feliciano, D. L. Graham, and P. P. Freita, "Effect of spin-valve sensor magnetostatic fields on nanobead detection for biochip applications," J. Appl. Phys., vol. 97, 2005.

[8] L. Ejsing, M. F. Hansen, A. K. Menon, H. A. Ferreira, D. L. Graham, and P. P. Freitas, "Planar Hall effect sensor for magnetic micro- and nanobead detection," Appl. Phys. Lett., vol. 84, p. 23, 2004.

[9] A. Garcia-Arribas, F. Martinez, E. Fernandez, I. Ozaeta, G. V. Kurlyandskaya, A. V. Svalov, J. Berganzo, and J. M. Barandiaran, "GMI detection of magnetic-particles concentration in continuous flow," Sensors Actuators, vol. A 172, pp. 103-108, March 2011.

[10] M. Denoual, M. Harnois, S. Saez, C. Dolabdjian, and V. Senez, "Microfluidic microsystem for magnetic sensing of nanoparticles with giant magneto-impedance technology," Transducers, 2011

[11] Kurlyandskaya, M. L. Sanchez, B. Hernando, V. M. Prida, P. Gorria, and M. Tejedor, "Giant-magnetoimpedance-based sensitive element as a model for biosensors," App. Phys. Lett. , vol. 82, 2003.

[12] H. Chiriac, M. Tibu, A. E. Moga, and D. D. Herea, "Magnetic GMI sensor for detection of biomolecules," J. Magnet. Magn. Mater., vol. 293, pp. 671-676, 2005.

[13] H. Yang, L. Chen, C. Lei, J. Zhang, D. Li, Z. Zhou, C. Bao, H. Hu, X. Chen, F. Cui, S. Zhang, Y. Zhou, and D. Cui, "Giant magnetoimpedance-based microchannel system for quick and parallel genotyping of human papilloma virus type 16/18," Appl. Phys. Lett., vol. $97,2012$.

[14] K. Taton, D. Johnson, P. Guire, E. Lange, and M. Tondra, "Lateral flow immunoassay using magnetoresistive sensors," J. Magnet. Magn. Mater., vol. 321, pp. 1679-1682, 2009

[15] G. Song, S. Xiangyang, R. B. James, M. Banaszak Holl, and G. O. Bradford, "Development of a remanence measurement-based SQUID system with in-depth resolution for nanoparticles imaging," Phys. Med. Biol., pp. 177-188, 2009

[16] D. Ménard and A. Yelon, "Theory of longitudinal magnetoimpedance in wires," J. Appl. Phys., vol. 88, pp. 379-393, 2000.

[17] MXT, Inc.. Montreal, QC, Canada [Online]. Available: http://www. mxt.ca

[18] V. Dousset, C. Gomez, K. G. Petry, C. Delalande, and J. M. Caille, "Dose and scanning delay using USPIO for central nervous system macrophage imaging," Elsevier Science, Magnetic Resonance Materials in Physics, Biology and Medicine, vol. 8, pp. 185-189, 1999.

[19] B. Dufay, S. Saez, C. Dolabdjian, A. Yelon, and D. Ménard, "Impact of electronic conditioning on the noise performance of a two-port network Giant MagnetoImpedance magnetometer," IEEE Sensors J., vol. 11, pp. 1317-1324, Nov. 2010 